\title{
The Phospho-Dependent Dynamin-Syndapin Interaction Triggers Activity-Dependent Bulk Endocytosis of Synaptic Vesicles
}

\author{
Emma L. Clayton, ${ }^{1}$ Victor Anggono, ${ }^{2}$ Karen J. Smillie, ${ }^{1}$ Ngoc Chau, ${ }^{2}$ Phillip J. Robinson, ${ }^{2}$ and Michael A. Cousin ${ }^{1}$ \\ ${ }^{1}$ Membrane Biology Group, Centre for Integrative Physiology, George Square, University of Edinburgh, Edinburgh EH8 9XD, United Kingdom, and \\ ${ }^{2}$ Children's Medical Research Institute, The University of Sydney, Wentworthville 2145, New South Wales, Australia
}

\begin{abstract}
Synaptic vesicles (SVs) are retrieved by more than one mode in central nerve terminals. During mild stimulation, the dominant SV retrieval pathway is classical clathrin-mediated endocytosis (CME). During elevated neuronal activity, activity-dependent bulk endocytosis (ADBE) predominates, which requires activation of the calcium-dependent protein phosphatase calcineurin. We now report that calcineurin dephosphorylates dynamin I in nerve terminals only above the same activity threshold that triggers ADBE. ADBE was arrested when the two major phospho-sites on dynamin I were perturbed, suggesting that dynamin I dephosphorylation is a key step in its activation. Dynamin I dephosphorylation stimulates a specific dynamin I-syndapin I interaction. Inhibition of this interaction by competitive peptides or by site-directed mutagenesis exclusively inhibited ADBE but did not affect CME. The results reveal that the phospho-dependent dynamin-syndapin interaction recruits ADBE to massively increase SV endocytosis under conditions of elevated neuronal activity.
\end{abstract}

\section{Introduction}

Neuronal activity results in the fusion of neurotransmitter containing synaptic vesicles (SVs) with the nerve terminal plasma membrane. After fusion, $\mathrm{SV}$ membrane is retrieved by at least two different endocytic modes. Clathrin-mediated endocytosis (CME) is the dominant mode of SV endocytosis during mild neuronal activity in the CNS, regenerating small SVs directly from the plasma membrane (Granseth et al., 2006). During increased neuronal activity, a second SV retrieval mode is triggered, called activity-dependent bulk endocytosis (ADBE) (Royle and Lagnado, 2003; Rizzoli and Betz, 2005; Wu et al., 2007; Clayton et al., 2008). ADBE immediately corrects for gross changes in nerve terminal surface area by the rapid formation of endosome-like structures direct from the plasma membrane. ADBE is strictly activity dependent and does not continue when stimulation terminates, whereas CME persists for minutes afterward (Clayton et al., 2008). Therefore, ADBE is the dominant membrane retrieval mode during elevated neuronal activity, whereas CME is dominant after stimulation.

CME and ADBE are likely to use similar or the same endocytic

Received April 27, 2009; accepted May 15, 2009.

This work was supported by grants from the Wellcome Trust (Ref: 070569 and 084277) and Epilepsy Research UK (0503) to M.A.C., and from the Australian National Health and Medical Research Council (NHMRC) to P.J.R. V.A. was supported by an Australian NHMRC Biomedical Overseas Fellowship (I.D. 477108). Dr. Alan Prescott and John James (both University of Dundee, Dundee, UK) are thanked for excellent technical assistance. The shRNA constructs were generated in Dr. Richard Huganir's laboratory (Johns Hopkins University School of Medicine, Baltimore, MD).

Correspondence should be addressed to Michael A. Cousin, Membrane Biology Group, Centre for Integrative Physiology, George Square, University of Edinburgh, Edinburgh EH8 9XD, UK. E-mail: m.cousin@ed.ac.uk.

V. Anggono's present address: Department of Neuroscience, Howard Hughes Medical Institute, The Johns Hopkins University School of Medicine, 725 North Wolfe Street, 1001 Hunterian, Baltimore, MD 21205.

DOI:10.1523/JNEUROSCI.1976-09.2009

Copyright $\odot 2009$ Society for Neuroscience $\quad$ 0270-6474/09/297706-12\$15.00/0 proteins; however, they exhibit distinct regulation by signal transduction pathways. For example, ADBE is strictly regulated by the calcium-dependent protein phosphatase calcineurin (Evans and Cousin, 2007; Clayton and Cousin, 2008). ADBE is activated coincident with stimulation, suggesting that its molecular trigger must sense increased neuronal activity. Calcineurin is an ideal candidate for the ADBE activity-dependent sensor, since it dephosphorylates at least eight endocytic proteins called the dephosphins on nerve terminal depolarization (Cousin and Robinson, 2001; Lee et al., 2005). It is currently unknown whether the dephosphorylation of either one, a group of, or all of the dephosphins mediates ADBE.

Dynamin I is a dephosphin whose GTPase activity is essential for SV endocytosis (Marks et al., 2001). It binds to a number of Src homology 3 (SH3) domain-containing proteins that have an $\mathrm{N}$-terminal bin-amphiphysin-RVS (BAR) domain, such as amphiphysin, endophilin, and syndapin (Grabs et al., 1997; Ringstad et al., 1997; Qualmann et al., 1999); however, only the syndapin I interaction is specifically stimulated by dynamin I dephosphorylation (Anggono et al., 2006). Both dynamin I dephosphorylation and its downstream interaction with syndapin are essential for SV endocytosis in central nerve terminals (Anggono et al., 2006; Anggono and Robinson, 2007), suggesting a key role for these events in the triggering of ADBE. In support, the dynamin I knock-out mouse exhibits a defect in SV endocytosis only when neuronal activity is elevated, with this defect only apparent during, but not after, stimulation (Ferguson et al., 2007). Since ADBE is the dominant SV endocytosis route during these stimulation conditions, this suggests a key role for the phosphorylation-dependent dynamin-syndapin interaction in the triggering of ADBE by elevated neuronal activity. 
Our main aim was to determine whether dynamin I dephosphorylation and its association with syndapin are specifically required for ADBE. We found that dynamin I dephosphorylation is a reporter of elevated neuronal activity and that perturbation of the dynamin-syndapin complex specifically blocked ADBE but not CME. Thus, ADBE is triggered by the activity-dependent dephosphorylation of dynamin I, which stimulates the formation of a dynamin-syndapin complex in central nerve terminals.

\section{Materials and Methods}

Materials. FM2-10, FM1-43, tetramethylrhodamine-dextran, penicillin/ streptomycin, phosphate-buffered salts, fetal calf serum, and minimal essential medium were obtained from Invitrogen. Dynamin I phosphospecific antibodies were described previously (Tan et al., 2003). shRNA against syndapin was designed using the pSUPER vector system (Oligoengine), using the following oligonucleotides: Oligo 1, 5'-GCGCCAGCTCATCGAGAAATTCAAGAGATTTCTCGATGAGCTGGCGC3'; Oligo 2, 5' -GCCAAGATCGAGAAGGCATACTTCAAGAGAGTATGCCTTCTCGATCTTGGC-3'. Synaptophysin antibody was from Synaptic Systems. Syndapin antibody was from BD Transduction Laboratories. Peptides were synthesized by Genemed Synthesis. Glutaraldehyde and osmium tetroxide were from Agar Scientific. Dynasore was a gift from Dr. A. McCluskey (University of Newcastle, Callaghan, NSW, Australia). All other reagents were from Sigma.

Primary cell culture, transfections, and immunofluorescence studies. Primary cultures of cerebellar granule neurons were prepared from the cerebella of 7-d-old Sprague Dawley rat pups as previously described (Tan et al., 2003). All dynamin I sequences were fused to the fluorescent protein mCerulean (Rizzo et al., 2004) at their C termini, since the fluorescence of this protein does not interfere with fluorescein optics (Anggono et al., 2006; Evans and Cousin, 2007). In the shRNA studies, empty mCerulean vector was coexpressed with the shRNA vector to mark transfected neurons. Transfections were performed using calcium phosphate precipitation (Tan et al., 2003). Immunofluorescence studies were performed as previously described (Tan et al., 2003). Syndapin expression was monitored by measuring the immunofluorescence intensity in the cell body of transfected neurons, since low transfection efficiency precludes analysis by Western blotting. Fluorescence intensity was expressed as a percentage of the untransfected neurons in the same field of view (after subtraction of background fluorescence). At least three independent experiments were performed, with at least three fields of view being assessed for each experiment.

Fluorescence imaging of SV turnover. Cultures were removed from culture medium and left for $10 \mathrm{~min}$ in incubation medium [in mM: 170 $\mathrm{NaCl}, 3.5 \mathrm{KCl}, 0.4 \mathrm{KH}_{2} \mathrm{PO}_{4}, 20$ TES ( $N$-tris[hydroxy-methyl]-methyl-2aminoethane-sulfonic acid), $5 \mathrm{NaHCO}_{3}, 5$ glucose, $1.2 \mathrm{Na}_{2} \mathrm{SO}_{4}, 1.2$ $\mathrm{MgCl}_{2}, 1.3 \mathrm{CaCl}_{2}, \mathrm{pH}$ 7.4)] Cultures were then mounted in a Warner imaging chamber (RC-21BRFS). Invaginating membrane was loaded with either FM2-10 $(100 \mu \mathrm{M})$ or FM1-43 $(10 \mu \mathrm{M})$ by evoking SV turnover with a train of 800 action potentials $(80 \mathrm{~Hz}, 100 \mathrm{~mA}, 1 \mathrm{~ms}$ pulse width, delivered using platinum wires embedded in the imaging chamber). Dye was washed from the cultures immediately after stimulation (S1 loading). After a $10 \mathrm{~min}$ rest period, accumulated dye was unloaded from nerve terminals using a $30 \mathrm{~s}$ stimulus of $50 \mathrm{~mm} \mathrm{KCl}(\mathrm{NaCl}$ removed to maintain osmolarity). This provides an estimate of the total number of SVs turned over during stimulation $(\Delta \mathrm{S} 1)$. Experiments were performed using a Zeiss TV-100 epifluorescence microscope and a $40 \times$ oil objective. Transfected neurons were visualized at $430 \mathrm{~nm}$ excitation, whereas FM1-43 and FM2-10 were visualized at $500 \mathrm{~nm}$ (both $>525 \mathrm{~nm}$ emission).

The protocol for studies with penetratin-tagged peptides was essentially the same with the exception that the $\mathrm{S} 1$ protocol was repeated after a 20 min rest period (S2 loading and unloading). Thus, for any selected nerve terminal, the $\Delta \mathrm{S} 2$ response has a matched individual internal control $(\Delta \mathrm{S} 1)$. Peptides $(30 \mu \mathrm{M})$ were added $15 \mathrm{~min}$ before and during S2 loading. These results are represented as either cumulative histograms ( $\Delta \mathrm{S} 2 / \Delta \mathrm{S} 1$ for individual nerve terminals) or averaged data (average $\Delta \mathrm{S} 2 /$ $\Delta \mathrm{S} 1)$. Dye unloading was visualized using a Nikon Diaphot-TMD epiflu-
A
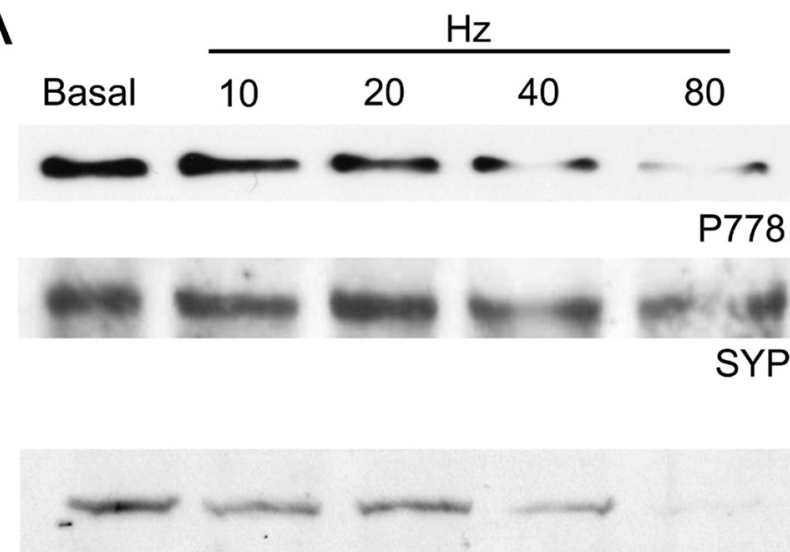

P774

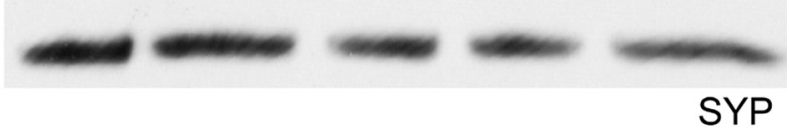

$B$

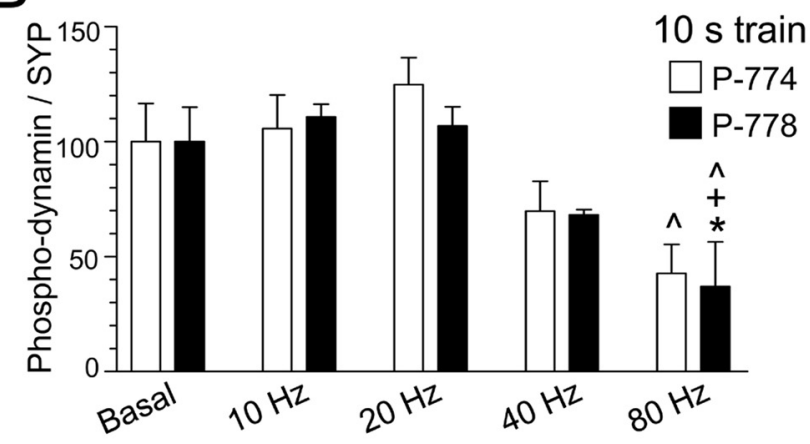

C

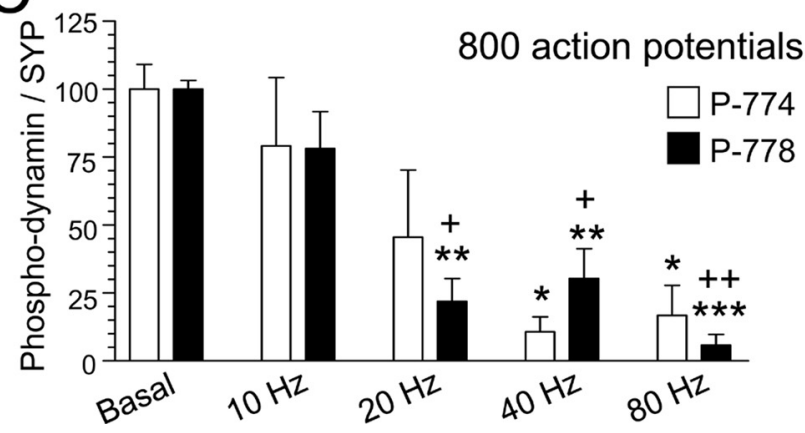

Figure 1. A-C, Dynamin I is dephosphorylated in an activity-dependent manner. Granule neuron cultures were subjected to action potential trains of increasing frequency $(10,20,40$, or $80 \mathrm{~Hz}$ ) with either a fixed duration (10 s) (B) or a fixed number (800 action potentials) (C). The extent of phosphorylation on either residue Ser-774 (open bars) or Ser-778 (solid bars) was assessed by Western blotting with phospho-specific antibodies. Synaptophysin (SYP) blots were performed as loading controls. $\boldsymbol{A}$ shows typical Western blots for phospho-dynamin I (P-778, P-774) and SYP. Results presented in $\boldsymbol{B}$ and $\boldsymbol{C}$ for phospho-dynamin I are corrected for protein level (SYP), and are normalized to control \pm SEM (10 s: P774, $n=4 ;$ P778, $n=3 ; 800$ action potentials: P774, $n=3 ; \mathrm{P778}, n=4)$. One-way ANOVA; ${ }^{*} p<0.05,{ }^{* *} p<0.01,{ }^{* * *} p<$ 0.001 to $\mathrm{Ctrl} ;{ }^{+} p<0.05,{ }^{++} p<0.01,10 \mathrm{~Hz} ; \wedge p<0.05,20 \mathrm{~Hz}$.

orescence microscope and $20 \times$ air objective at $480 \mathrm{~nm}$ excitation and $>510 \mathrm{~nm}$ emission.

For both sets of experiments fluorescent images were visualized using a Hamamatsu Orca-ER CCD digital camera and off-line imaging software (Simple PCI, Compix). Nerve terminals were selected by their ability to load and unload dye on stimulation. Regions of interest of identical 


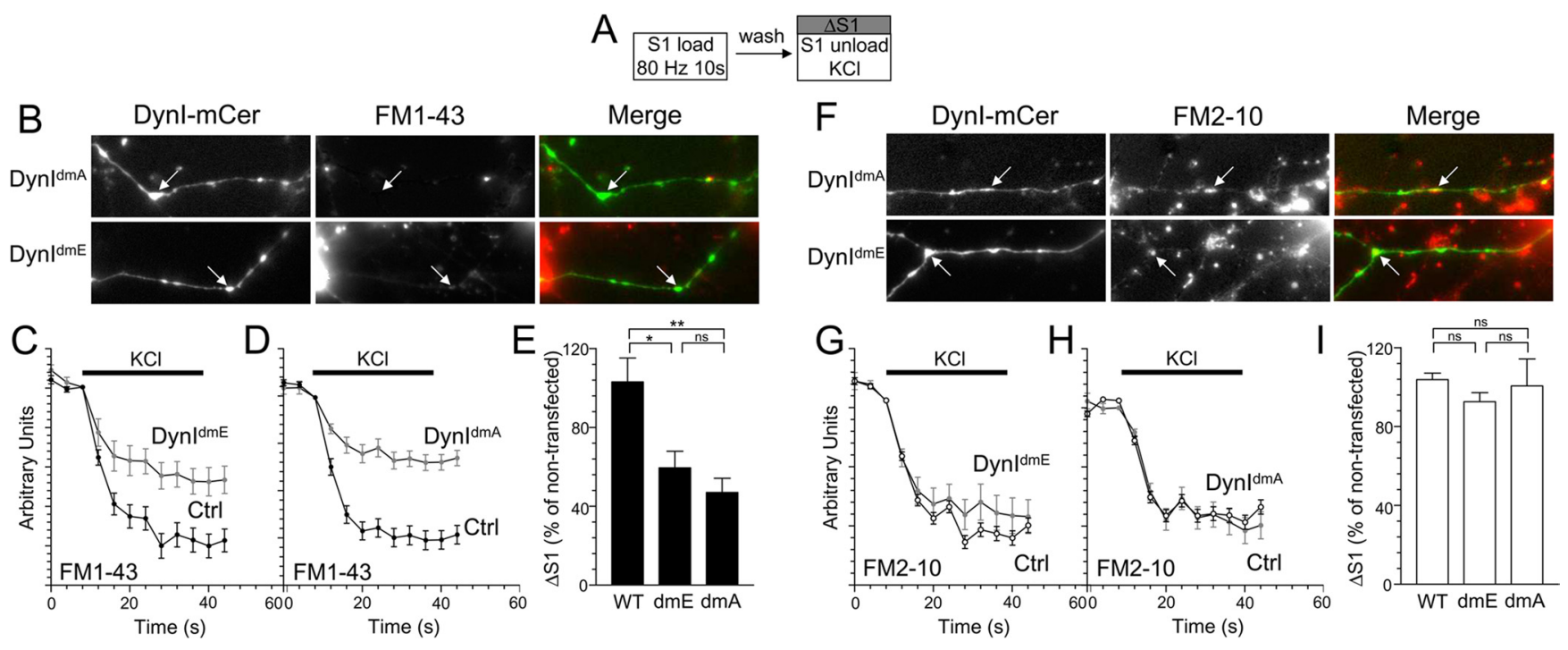

Figure 2. Dynamin I dephosphorylation is required for FM1-43, but not FM2-10 uptake. $A$, Granule neuron cultures were loaded and unloaded with FM dyes using the protocol displayed. Dyes were loaded with 800 action potentials $(80 \mathrm{~Hz}$ ) and then washed away immediately. Dye unloading was stimulated by a 30 s stimulus of $50 \mathrm{~mm} \mathrm{KCl}$. $\boldsymbol{B}$, Left panels display transfected neurons (either Dynl $^{\mathrm{dmA}}$-mCer or Dynl ${ }^{\mathrm{dmE}}$-mCer), middle panels display FM1-43 loading, and right panels display a merged image of transfected neurons (green) and FM1-43 loading (red). Arrows indicate nerve terminals. C, D, Time course of FM1-43 unloading from either transfected (gray) or nontransfected (black) nerve terminals in the same field of view $\left(\boldsymbol{C}\right.$, Dynl ${ }^{\mathrm{dmE}}$-mCer; or $\boldsymbol{D}$, Dynl $\left.{ }^{\mathrm{dmA}}-\mathrm{mCer}\right) . \boldsymbol{E}$, Bar graph displays the extent of FM1-43 loading of transfected nerve terminals as a percentage of untransfected nerve terminals in the same field of view $\left(\Delta \mathrm{S} 1 \pm \mathrm{SEM}\right.$; Dynl ${ }^{\mathrm{WT}}$-mCer, $n=3$; Dynl $^{\text {dmE-}}$-mCer and Dynl ${ }^{\mathrm{dmA}}$-m(er, $n=4$ ). $\boldsymbol{F}$, Left panels display transfected neurons (either Dynl ${ }^{\text {dmA }}$-m(er or Dynl ${ }^{\text {dmE}}$-m(er), middle panels display FM2-10 loading, and right panels display a merged image of transfected neurons (green) and FM2-10 loading (red). Arrows indicate nerve terminals. $\boldsymbol{G}, \boldsymbol{H}$, Time course of FM2-10 unloading from either transfected (gray) or nontransfected (black) nerve terminals in the same field of view (G, Dynl ${ }^{\mathrm{dmE}}$-mCer; or $\boldsymbol{H}$, Dynl ${ }^{\mathrm{dmA}}$-mCer). $\boldsymbol{I}$, Bar graph displays the extent of FM2-10 loading of transfected nerve terminals as a percentage of untransfected nerve terminals in the same field of view ( $\Delta \mathrm{S} 1 \pm \mathrm{SEM}$; Dynl ${ }^{\mathrm{WT}}$-mCer, $n=3$; Dynl ${ }^{\mathrm{dmE}}$-mCer, $n=4$; Dynl ${ }^{\mathrm{dmA}}$-mCer, $\left.n=5\right)$. One-way ANOVA: ${ }^{*} p<0.05$, ${ }^{* *} p<0.01$.
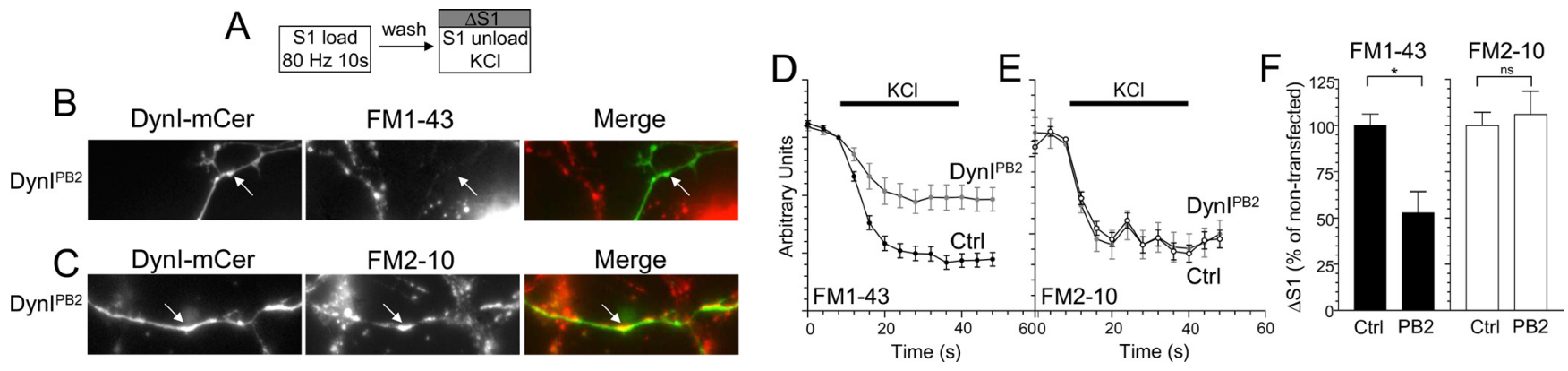

Figure 3. The dynamin-syndapin interaction is required for FM1-43 but not FM2-10 uptake. $A$, Granule neuron cultures were loaded and unloaded with FM dyes using the protocol displayed. Dyes were loaded with 800 action potentials $(80 \mathrm{~Hz})$ and then washed away immediately. Dye unloading was stimulated by a $30 \mathrm{~s} \mathrm{stimulus} \mathrm{of} 50 \mathrm{~mm} \mathrm{KCl}$. B, C, Left panels display transfected neurons (Dynl $^{\mathrm{PB} 2}$-m(er), middle panels display either FM1-43 (B) or FM2-10 (C) loading, and right panels display a merged image of transfected neurons (green) and FM1-43 loading (red). Arrows indicate nerve terminals. $\boldsymbol{D}, \boldsymbol{E}$, Time course of either FM1-43 (D) or FM2-10 (E) unloading from either transfected (gray) or nontransfected (black) nerve terminals in the same field of view is displayed. Bar graph displays the extent of either FM1-43 (black bars) or FM2-10 (open bars) loading of transfected nerve terminals (PB2) compared with untransfected nerve terminals (Ctrl) in the same field of view $(\Delta S 1 \pm$ SEM; FM1-43, $n=3 ; \mathrm{FM} 2-10, n=4)$. Student's $t$ test, ${ }^{*} p<0.05$.

size were placed over nerve terminals and the total fluorescence intensity of these regions was monitored over time. Fluorescence time courses from individual nerve terminals were aligned to the point before stimulation and averaged. The total fluorescence decrease was expressed as a percentage of untransfected nerve terminals for overexpression studies, or as a $\Delta \mathrm{S} 2 / \Delta \mathrm{S} 1$ ratio with control nerve terminals for peptide studies. For overexpression studies, at least 5 nerve terminals per transfected neuron and at least 50 untransfected nerve terminals were selected per field of view. For peptide studies at least 70 nerve terminals were selected per field of view. Fields of view were observed from at least three independent experiments from at least two different groups of cultures. Cultures were used routinely between 8 and 10 days in vitro.

Fluorescence imaging of dextran uptake. The uptake of tetramethylrhodamine-dextran $(40 \mathrm{kDa})$ was monitored as described previously (Clayton et al., 2008). Briefly, cultures were removed from culture medium, left for $10 \mathrm{~min}$ in incubation medium, and then stimulated with a train of 800 action potentials $(80 \mathrm{~Hz}, 10 \mathrm{~s})$.
Tetramethylrhodamine-dextran $(50 \mu \mathrm{M})$ was present during the stimulus and was washed away immediately after stimulation. The extent of loading was determined by the number of fluorescent puncta in a defined field of view $(130 \mu \mathrm{m} \times 130 \mu \mathrm{m})$ using a $20 \times$ air objective at $550 \mathrm{~nm}$ excitation and $>575 \mathrm{~nm}$ emission. Thresholding analysis was performed to discount regions too large to represent individual nerve terminals (diameter $>2 \mu \mathrm{m}$ ). Greater than $90 \%$ of dextran puncta colocalize with nerve terminals (assessed using coloading with FM1-43; data not shown). The average number of dextran puncta per field for each experiment (usually 10 fields of view per experiment) were averaged for the same conditions and subtracted from background fluorescence. The final value for dextran puncta was obtained by averaging the individual averages from three independent experiments (all with 10 fields of view). To ensure the density of nerve terminals was consistent between fields and experimental conditions, experiments were performed on the same set of cultures. Cultures were used between 8 and 10 days in vitro. Experiments using neurons transfected with syndapin shRNA were performed in the 
A
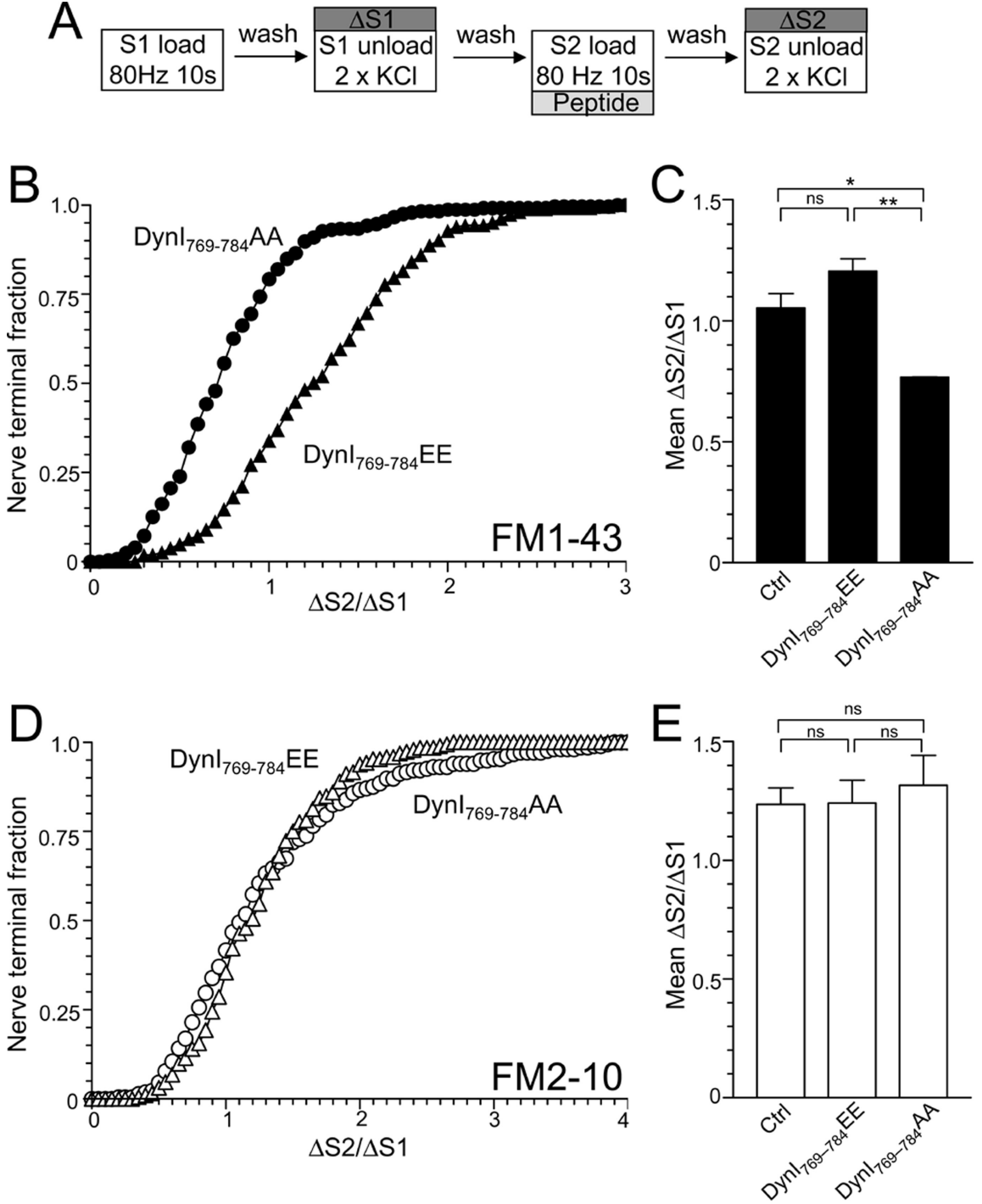

Figure 4. Dynamin I phospho-deficient peptide, but not phospho-mimetic peptides, block FM1-43 uptake. $A$, Granule neuron cultures were loaded and unloaded with FM dyes using the protocol displayed. Dyes were loaded at both S1 and S2 with 800 action potentials $\left(80 \mathrm{~Hz}\right.$ ) and then washed away immediately. At S2, cultures were preincubated with $30 \mu \mathrm{m}$ peptide (either Dynl ${ }_{769-}$ ${ }_{784} \mathrm{AA}$ or Dynl ${ }_{769-784} \mathrm{EE}$ ) for $15 \mathrm{~min}$ before loading. At both $\mathrm{S} 1$ and S2, unloading was stimulated by two 30 s stimuli of $50 \mathrm{~mm} \mathrm{KCl}$. $\boldsymbol{B}, \boldsymbol{D}$, Cumulative histograms of the effect of peptide on either FM1-43 (B) or FM2-10 (D) unloading in individual nerve terminals $(\Delta S 2 / \Delta S 1)$ are displayed (circles represent Dynl ${ }_{769-784} A A$, whereas triangles represent Dynl $\left.{ }_{769-784} \mathrm{EE}\right)$. Bar graphs in $\boldsymbol{C}$ and $\boldsymbol{E}$ display the mean $\Delta S 2 / \Delta S 1$ response \pm SEM in the absence (Ctrl) or presence of the peptides (all $n=3 \pm$ SEM except FM1-43 (trl, $n=4)$. One-way ANOVA; ${ }^{*} p<0.05,{ }^{* * *} p<0.001$.

same manner, with the number of dextran puncta per neuron calculated. At least three independent experiments were performed, with at least three neurons assessed for each experiment.

Labeling of endocytosis pathways by HRP. Cultures were processed for electron microscopy as previously described (Evans and Cousin, 2007; Clayton et al., 2008). Briefly, cultures were removed from culture medium into incubation medium, and after a 10 min rest period, cultures were stimulated with either $50 \mathrm{~mm} \mathrm{KCl}$ or 800 action potentials $(80 \mathrm{~Hz})$. HRP $(10 \mathrm{mg} / \mathrm{ml})$ was present during stimulation and washed away immediately afterward. Neurons were fixed in a $2 \%$ solution of glutaraldehyde in PBS for $30 \mathrm{~min}$ at $37^{\circ} \mathrm{C}$. After washing with $100 \mathrm{~mm}$ Tris, $\mathrm{pH} 7.4$, cultures were exposed to $0.1 \%$ diaminobenzidine and $0.2 \% \mathrm{H}_{2} \mathrm{O}_{2}$ in 100 mM Tris. On development of color, cultures were washed with $100 \mathrm{~mm}$ Tris and then stained with $1 \%$ osmium tetroxide for $30 \mathrm{~min}$. After washing, cultures were poststained with $2 \%$ uranyl acetate for $15 \mathrm{~min}$ and then dehydrated, using ethanol series and polypropylene oxide, and embedded, using Durcupan. Samples were sectioned, mounted on grids, and viewed using an FEI Tecnai 12 transmission electron microscope. Nerve terminals that contained HRP were analyzed and intracellular structures that were $<100 \mathrm{~nm}$ in diameter were arbitrarily designated to be SVs, whereas larger structures were designated to be endosomes.

Assays of dynamin I dephosphorylation. Cultures were removed from culture medium after a 10 min rest period in incubation medium and were then stimulated with trains of action potentials. Trains had either a fixed duration (10 s) or number (800 action potentials). Immediately after stimulation, SDS sample buffer (67 mM SDS, 2 mM EGTA, 9.3\% glycerol, 12\% $\beta$-mercaptoethanol, bromophenol blue, $67 \mathrm{~mm}$ Tris, $\mathrm{pH}$ 7.4) was added to lyse the neurons. Lysate was quickly removed and boiled for subsequent analysis by SDS-PAGE and Western blotting. The intensity of signal from phosphodynamin blots was normalized against the amount of synaptophysin and expressed as a percentage of control.

\section{Results}

Dynamin I is dephosphorylated in an activity-dependent manner

Many studies have shown that stimulusdependent dynamin I dephosphorylation occurs when nerve terminals are depolarized (Cousin and Robinson, 2001). In these studies, the extent of dephosphorylation was large and its speed was extremely rapid $(<2 \mathrm{~s})$. Such studies used either elevated $\mathrm{KCl}$ or ionomycin, which are strong stimuli that would be expected to activate both $\mathrm{CME}$ and $\mathrm{ADBE}$. In contrast, dynamin I dephosphorylation in response to action potential stimulation has never been investigated. This is critical, since stimulus intensity is a key determinant in the activity-dependent selection between multiple SV endocytosis modes. Hence our first aim was to determine whether dynamin I dephosphorylation occurred during both mild neuronal activity (when only CME is active) and elevated neuronal activity (which triggers both ADBE and CME).

We challenged neuronal cultures with trains of action potentials of increasing intensity and examined the calcineurin-dependent dephosphorylation of dynamin I. At rest, dynamin I is phosphorylated at Ser-774 and Ser-778 in the phospho-box region of its proline-rich $\mathrm{C}$ terminus (Tan et al., 2003; Graham et al., 2007). When cultures were challenged with 800 action potentials at $80 \mathrm{~Hz}$ [a protocol that maximally stimulates ADBE (Clayton et al., 2008)], dynamin I was dephosphorylated at both phospho-sites to a similar extent (Fig. 1). When the level of activity was reduced by decreasing stimulation frequency (and keeping train duration constant), the extent of dephosphorylation decreased until no dephosphorylation was observed during stimulation with either 200 or $100 \mathrm{ac}$ tion potentials $(20 \mathrm{~Hz}$ and $10 \mathrm{~Hz}$, respectively) (Fig. $1 \mathrm{~B}$ ). Thus, dynamin I dephosphorylation at these twin sites is activity dependent and has an activation threshold in nerve terminals.

To ascertain whether the pattern of neuronal activity could also regulate dynamin I dephosphorylation, action potential 
trains of the same number but different stimulation frequency were applied. A marked frequency-dependent dynamin I dephosphorylation was again observed. This ranged from no effect with 800 action potentials at $10 \mathrm{~Hz}$, to a very strong dephosphorylation with the same number of action potentials at $80 \mathrm{~Hz}$ (Fig. 1C). The results show that calcineurin does not dephosphorylate dynamin I during mild neuronal activity, conditions in which CME is the dominant mode of SV endocytosis in nerve terminals (Granseth et al., 2006). Rather, activity-dependent dynamin I dephosphorylation is tuned to the same levels of activity previously reported to trigger ADBE.

\section{Dynamin I dephosphorylation is required for $\mathrm{ADBE}$}

Our next aim was to determine whether there is a role for the two dynamin I phospho-sites in ADBE. We examined the effect of interfering with the dynamin I phosphorylation cycle through overexpression of either phospho-deficient or phospho-mimetic mutants of full-length dynamin I in neuronal cultures. Dynamin I mutants were generated by the double mutation $(\mathrm{dm})$ of both Ser-774 and Ser778 to either alanine (phospho-deficient, DynI ${ }^{\mathrm{dmA}}$-mCer) or glutamate (phospho-mimetic, DynI ${ }^{\mathrm{dmE}}$-mCer), respectively (supplemental Fig. 1A, available at www.jneurosci.org as supplemental material). When previously overexpressed in neuronal cultures, both dominant-negative mutants inhibited the loading, but not the unloading, of the styryl dye FM4-64, demonstrating a selective block of SV endocytosis (Anggono et al., 2006). However, the dissection of CME from ADBE requires two different styryl dyes: FM1-43 and FM2-10. FM1-43 dye uptake labels both CME and ADBE in neurons, whereas FM2-10 only labels CME (Richards et al., 2000; Clayton and Cousin, 2008). Therefore, if these two phospho-sites are involved in ADBE, then the dominant-negative mutants would be expected to arrest the uptake of FM1-43 but not FM2-10.

Loading of FM1-43 was examined in cultures overexpressing either DynI ${ }^{\mathrm{dmA}}$-mCer or $\mathrm{DynI}^{\mathrm{dmE}}$-mCer stimulated by $800 \mathrm{ac}-$ tion potentials $(80 \mathrm{~Hz})$ to maximally activate $\mathrm{ADBE}$ (Clayton et al., 2008). A robust inhibition of FM1-43 loading was observed with either mutant (Fig. 2A-E). In contrast, overexpression of full-length wild-type dynamin I (DynI ${ }^{\mathrm{WT}}$-mCer) did not affect FM1-43 uptake (Fig. 2E). These results with FM1-43 are essentially the same as those previously reported for FM4-64 (Anggono et al., 2006), including no effect of the mutants on the kinetics of dye unloading, an indicator of SV exocytosis (supplemental Fig. 2, available at www.jneurosci.org as supplemental material). In contrast to the inhibition of FM1-43 loading, DynI $^{\mathrm{dmA}}$-mCer or DynI ${ }^{\mathrm{dmE}}$-mCer overexpression had no effect on FM2-10 uptake or unloading (Fig. 2F-I; supplemental Fig. 2, available at www.jneurosci.org as supplemental material). Since phospho-site-specific mutants of dynamin I inhibit the uptake of FM1-43 but not FM2-10 under identical conditions in neuronal culture, the results suggest that the dynamin I phospho-box is specifically involved in ADBE.

\section{Activity-dependent dynamin-syndapin interaction is required for $\mathrm{ADBE}$ but not $\mathrm{CME}$}

Dynamin I dephosphorylation stimulates an interaction with syndapin I in vitro and in intact nerve terminals (Anggono et al., 2006). The finding that dynamin I dephosphorylation is required for ADBE suggests that its interaction with syndapin may also be important. We therefore overexpressed dominant-negative fulllength dynamin I mutants that do not bind syndapin but that interact normally with amphiphysin and endophilin (DynI ${ }^{\mathrm{PB} 2}$ mCer) (supplemental Fig. $1 A$, available at www.jneurosci.org as supplemental material) (Anggono and Robinson, 2007). DynI ${ }^{\mathrm{PB} 2}$-mCer overexpression inhibited FM1-43 uptake evoked by 800 action potentials (Fig. $3 B, D, F$ ). The extent of inhibition was similar to that observed with the dynamin I phosphomutants, again with no effect on SV exocytosis (Fig. 2; supplemental Fig. 2, available at www.jneurosci.org as supplemental material). In contrast, FM2-10 loading was unaffected (Fig. $3 C, E, F)$. This selective inhibition of FM1-43 uptake suggests that the dynamin-syndapin interaction is required for ADBE.

An independent approach to disrupting the dynamin-syndapin interaction is via introduction of peptides into neurons that specifically block this interaction, but not that of dynamin I binding to amphiphysin or endophilin. These peptides correspond to the sequence of the dynamin I phospho-box (amino acids 769-784, incorporating Ser-774 and Ser-778) (supplemental Fig. $1 B$, available at www.jneurosci.org as supplemental material). When the two serines are replaced with alanine (Ser 774/778 to Ala, DynI $\mathrm{I}_{769-784} \mathrm{AA}$ ), such phospho-box peptides bind syndapin in cells and block the dynamin-syndapin interaction in vivo (Anggono et al., 2006). However the corresponding phospho-mimetic substitutions (Ser 774/778 to Glu, DynI ${ }_{769-784} \mathrm{EE}$ ) have no effect, since they do not bind syndapin I (Anggono et al., 2006). Both peptides were delivered into cultures using a penetratin delivery system (Cousin 

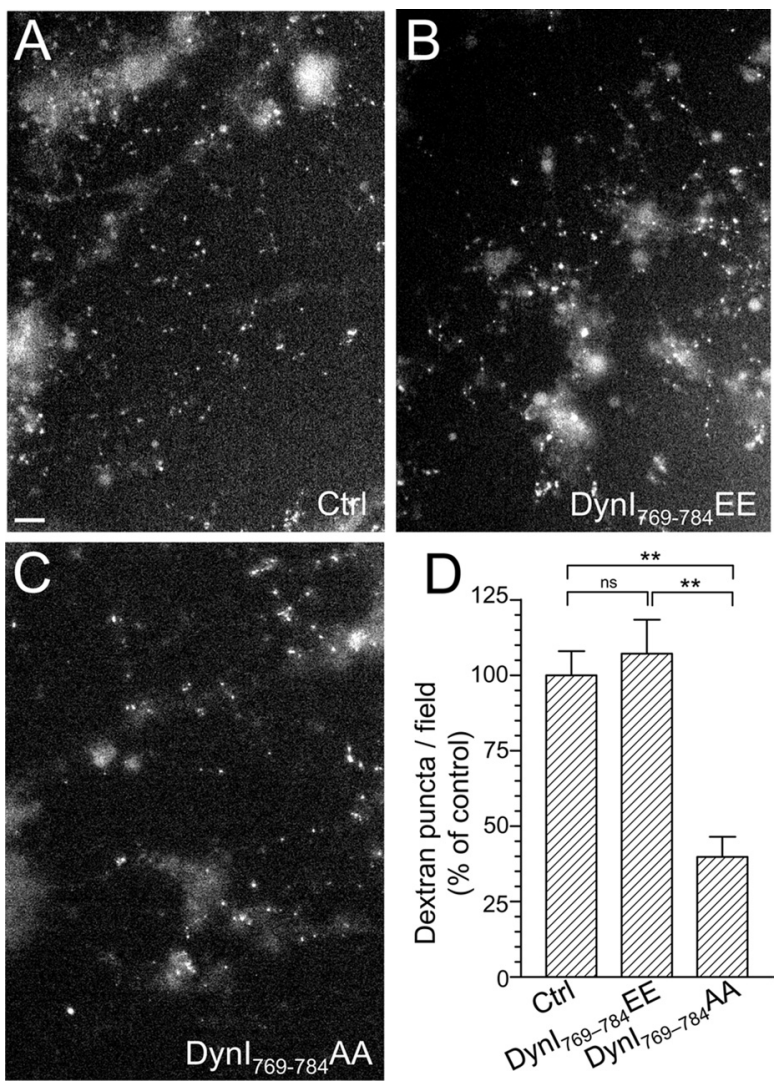

Figure 6. Dynamin I phospho-deficient peptide, but not phospho-mimetic peptide, blocks the uptake of large dextrans. Granule neuron cultures were incubated with $50 \mu \mathrm{m}$ tetramethylrhodamine-dextran and loading was stimulated by a train of 800 action potentials $(80 \mathrm{~Hz})$ followed by immediate dextran washout. Where indicated, cultures were incubated with $30 \mu \mathrm{m}$ dynamin peptides $15 \mathrm{~min}$ before and during stimulation (either Dynl ${ }_{769-784} \mathrm{AA}$ or Dynl $\left._{769-784} \mathrm{EE}\right)$. A-C, Panels show dextran loading in typical fields of view either in the absence of peptide (Ctrl) $(\boldsymbol{A})$ or in the presence of either Dynl ${ }_{769-784} \mathrm{EE}(\boldsymbol{B})$ or Dynl ${ }_{769-784} \mathrm{AA}(\boldsymbol{C})$. Scale bar represents $15 \mu \mathrm{m}$ in all images. $\boldsymbol{D}$, The mean number of dextran puncta per field of view as a percentage of control is displayed (all $n=3 \pm$ SEM, ${ }^{* *} p<0.01$, one-way ANOVA).

et al., 2003; Anggono et al., 2006), and their effect on both FM1-43 and FM2-10 uptake evoked by elevated neuronal activity ( 800 action potentials at $80 \mathrm{~Hz}$ ) was determined. An S2/S1 protocol was used, which allowed the effect of peptides to be directly compared with the control response from the same nerve terminals (Fig. 4A) (Evans and Cousin, 2007). When FM1-43 was examined, the phospho-deficient $\mathrm{DynI}_{769-784} \mathrm{AA}$ peptide inhibited loading, whereas the phospho-mimetic DynI ${ }_{769-784}$ EE peptide had no significant effect (Fig. $4 B, C$ ). The inhibition of loading by the DynI ${ }_{769-784} \mathrm{AA}$ peptide was not attributable to nonspecific effects on exocytosis, since it had no effect on FM1-43 unloading when present during the S2 unloading stimulus (data not shown). When FM2-10 uptake was examined, neither peptide had any effect (Fig. $4 D, E$ ). Thus the activity-dependent dynamin-syndapin interaction is essential for ADBE, since either overexpression of syndapin interaction mutants or the delivery of competitive peptides arrests ADBE in central nerve terminals.

The next aim was to determine whether the dynamin-syndapin complex was also required for CME, which is the dominant SV retrieval mode in nerve terminals during mild neuronal activity (Granseth et al., 2006). Since dynamin I is not dephosphorylated by mild neuronal activity (Fig. 1), it should not form a complex with syndapin under these conditions. Therefore, we tested whether the phospho-box peptides fail to alter SV endocytosis evoked by mild neuronal activity. FM1-43 uptake was evoked by 200 action potentials delivered at $10 \mathrm{~Hz}$, a protocol that activates CME, with no contribution from ADBE (Evans and Cousin, 2007; Clayton et al., 2008). Neither phospho-box peptide inhibited FM1-43 uptake evoked by mild stimulation (Fig. 5) [in contrast to the effect of the DynI ${ }_{769-784}$ AA peptide during intense stimulation (Fig. 4)]. Thus, there is an activity-dependent requirement for the dynamin-syndapin interaction in SV endocytosis, indicating a specific involvement in ADBE rather than CME.

Another form of CME that does not require a phosphoregulated dynamin I-syndapin I interaction is the classical receptor-mediated endocytosis pathway, such as transferrin receptor internalization. In agreement, neither of the phospho-box peptides affected transferrin endocytosis in U2OS cells at concentrations up to 10 -fold higher than that used with neuronal cultures (supplemental Fig. 3, available at www.jneurosci.org as supplemental material). Thus, the dynamin-syndapin interaction is not required for receptor-mediated endocytosis, supporting its unique activity-dependent role in $\mathrm{ADBE}$.

To independently confirm the specific role of the dynaminsyndapin interaction in ADBE, we next used a selective assay of
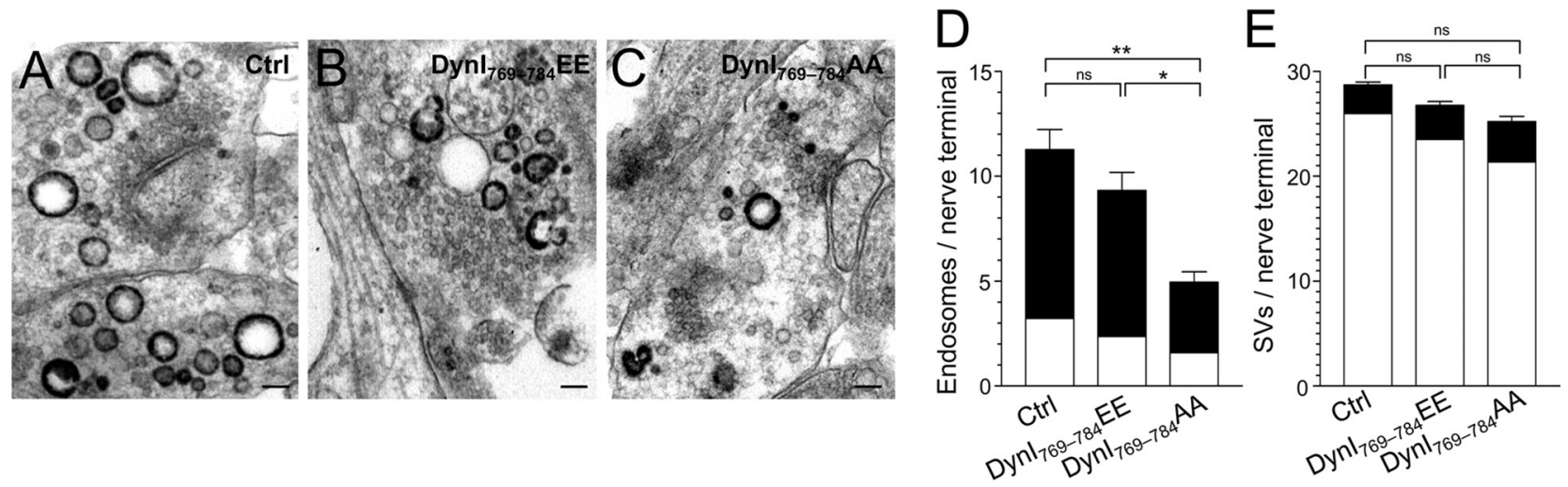

Figure 7. Dynamin I phospho-deficient peptide blocks HRP uptake into endosomes but not SVs. Granule neuron cultures were incubated with HRP and its loading was stimulated by a 2 min stimulus of $50 \mathrm{~mm} \mathrm{KCl}$. Where indicated, cultures were incubated with $30 \mu \mathrm{m}$ dynamin I peptides 15 min before and during stimulation (either Dynl ${ }_{769-784} \mathrm{AA}$ or Dynl $\left.\mathrm{769}_{-784} \mathrm{EE}\right)$. $A-C$, Panels show HRP-labeled structures in typical fields of view either in the absence of peptide $(\boldsymbol{A})$ or in the presence of either Dynl ${ }_{769-784} \mathrm{EE}(\boldsymbol{B})$ or Dynl $_{769-784} \mathrm{AA}(\boldsymbol{C})$. Scale bar represents $150 \mathrm{~nm}$ in all images. $\boldsymbol{D}$, $\boldsymbol{E}$, Mean number of either HRP-labeled (solid bars) or clear (open bars) endosomes (D) or SVs $(\boldsymbol{E})$ per nerve terminal is displayed either in the absence (Ctrl) or presence of peptides (Ctrl, $n=44$ nerve terminals; Dynl ${ }_{769-784} \mathrm{EE}, n=31$; Dynl ${ }_{769-784} \mathrm{AA}, n=23 ;$ all \pm SEM, ${ }^{* *} p<0.01,{ }^{*} p<0.05$, one-way ANOVA). 

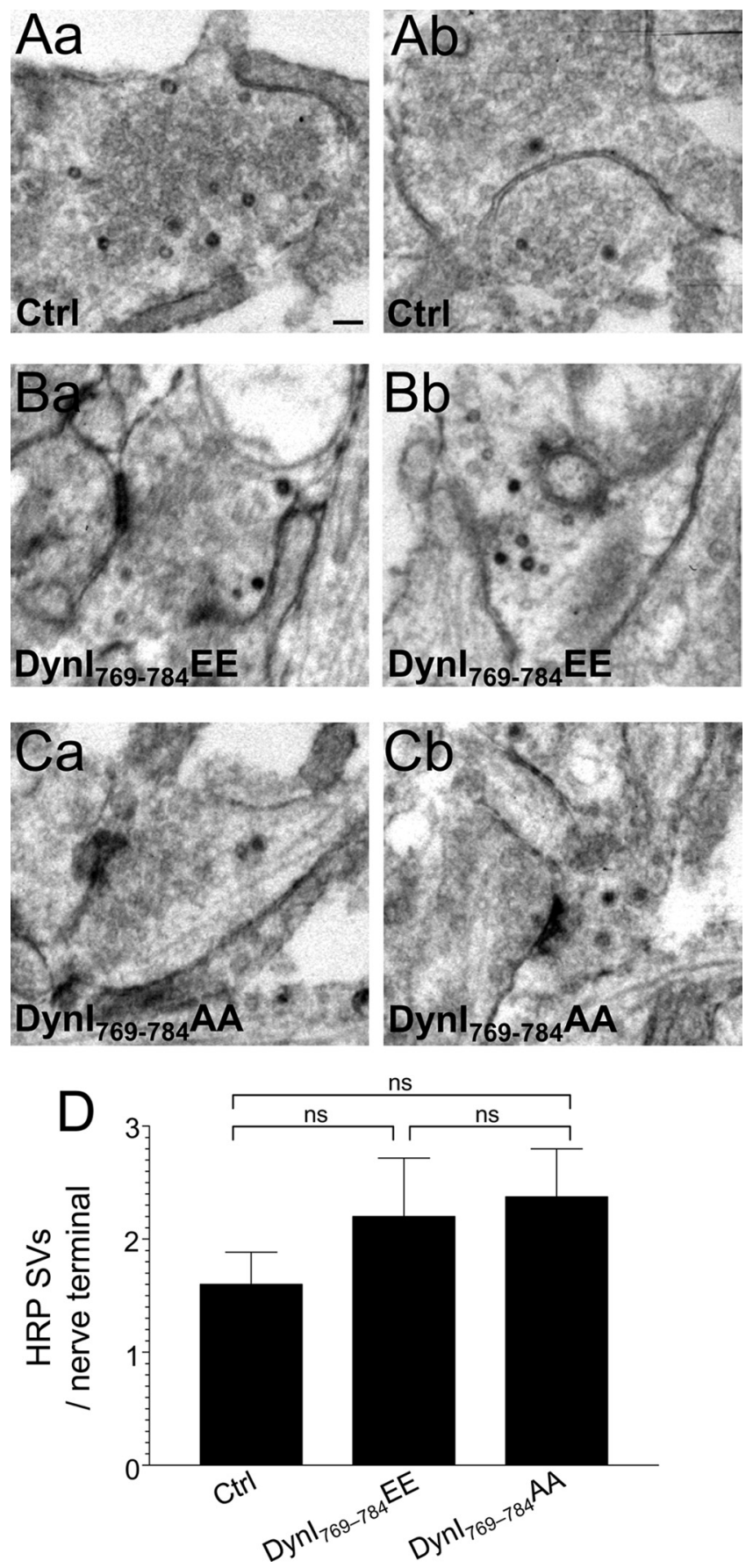

Figure 8. Dynamin I phospho-deficient peptide does not block HRP uptake into SVs during mild neuronal activity. Granule neuron cultures were incubated with HRP and loading was stimulated by a $20 \mathrm{~s}$ stimulus of 200 action potentials $(10 \mathrm{~Hz})$. Where indicated, cultures were incubated with $30 \mu \mathrm{m}$ dynamin I peptide (either Dynl ${ }_{769-784} \mathrm{AA}$ or Dynl ${ }_{769-784} \mathrm{E}$ ) 15 min before and during stimulation. $A-C$, Panels show HRP-labeled structures in typical fields of view either in the absence of peptide $(\boldsymbol{A} \boldsymbol{a}, \boldsymbol{A} \boldsymbol{b})$ or in the presence of either Dynl ${ }_{769-784} \mathrm{EE}(\boldsymbol{B} \boldsymbol{a}, \boldsymbol{B} \boldsymbol{b})$ or Dynl $_{769-784} \mathrm{AA}(\mathbf{C} \boldsymbol{a}, \boldsymbol{C b})$. Scale bar represents $250 \mathrm{~nm}$ in all images. $\boldsymbol{D}$, Mean number of HRPlabeled SVs per nerve terminal is displayed either in the absence (Ctrl) or presence of peptides (Ctrl, $n=49$ nerve terminals; Dynl ${ }_{769-784} \mathrm{EE}_{1} n=14$; Dynl ${ }_{769-784} \mathrm{AA}, n=23$; all \pm SEM, one-way ANOVA).

ADBE (since FM1-43 reports both CME and ADBE). The large $(40 \mathrm{kDa})$ fluorescent dextran tetramethylrhodamine-dextran selectively labels ADBE when applied during action potential trains since it is too large to enter single SVs (Holt et al., 2003; Teng et al., 2007; Clayton et al., 2008). Dextran uptake has previously
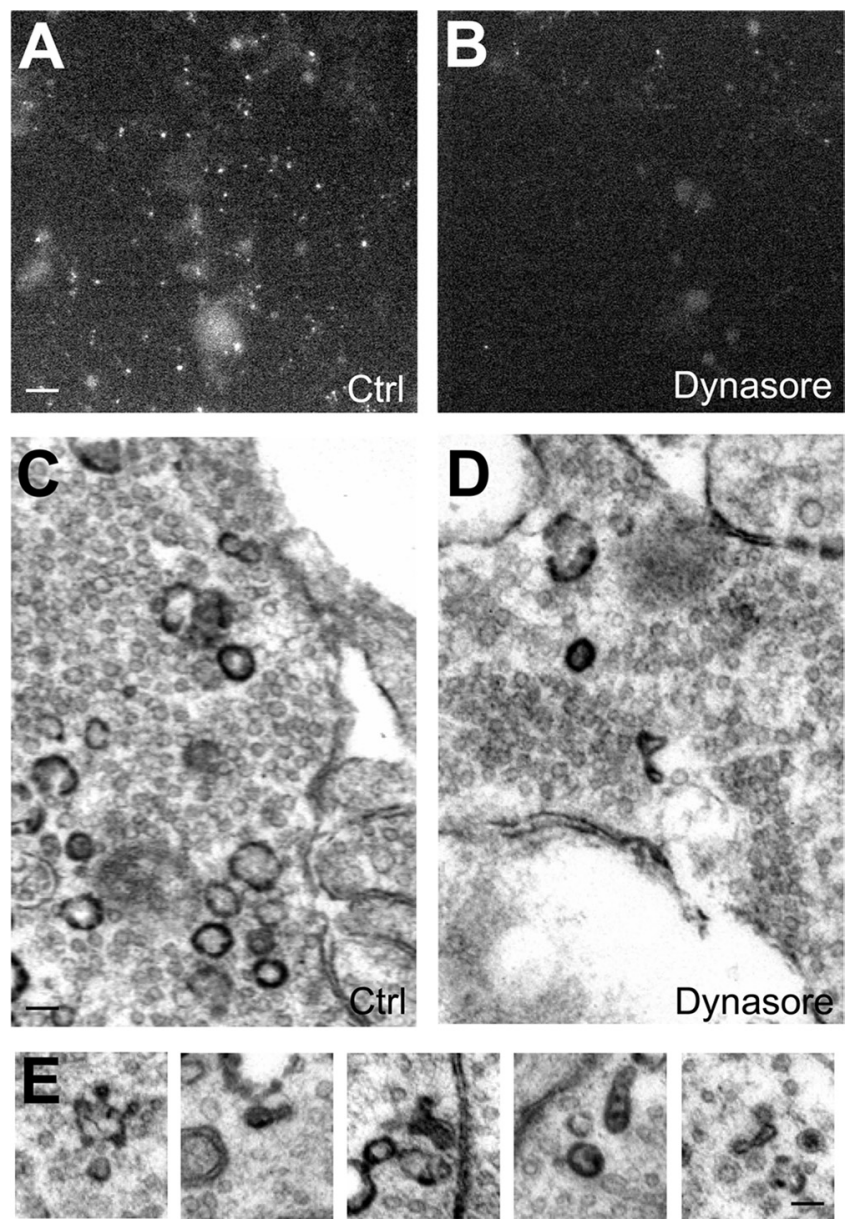

Figure 9. Dynamin GTPase activity is required for ADBE. Granule neuron cultures were incubated with $50 \mu \mathrm{m}$ tetramethylrhodamine-dextran and loading was stimulated by a train of 800 action potentials $(80 \mathrm{~Hz})$ followed by immediate dextran washout. Cultures were incubated with or without $80 \mu \mathrm{m}$ dynasore for $15 \mathrm{~min}$ before and during stimulation. $A-D$, Panels show dextran loading in typical fields of view either in the absence $(C \operatorname{crl})(\boldsymbol{A})$ or presence $(\boldsymbol{B})$ of dynasore. Scale bar represents $15 \mu \mathrm{m}$ in all images. $\boldsymbol{C}, \boldsymbol{D}$, Cultures were incubated with HRP and its loading was stimulated with 800 action potentials $(80 \mathrm{~Hz}$ ) followed by immediate HRP washout and fixation. Cultures were incubated with or without $80 \mu \mathrm{m}$ dynasore $15 \mathrm{~min}$ before and during stimulation. $\boldsymbol{C}-\boldsymbol{E}$, Panels show HRP-labeled structures in typical fields of view either in the absence $(\boldsymbol{C} \mathrm{trl})(\boldsymbol{C})$ or presence $(\boldsymbol{D})$ of dynasore. $\boldsymbol{E}$, Representative images of malformed HRP-labeled endosomes in the presence of dynasore. Scale bar: $(\boldsymbol{C}, \boldsymbol{D}), 150 \mathrm{~nm} ;(\boldsymbol{E}), 100 \mathrm{~nm}$.

been observed only during elevated but not mild neuronal activity in neuronal culture (Clayton et al., 2008). Addition of the DynI $_{769-784}$ EE phospho-box peptide did not affect dextran uptake evoked by 800 action potentials (Fig. $6 B, D$ ). In contrast, the DynI $_{769-784} \mathrm{AA}$ peptide produced a robust inhibition (Fig. 6C,D). Thus, inhibition of the activity-dependent dynamin-syndapin interaction arrests $\mathrm{ADBE}$ in central nerve terminals.

Parallel analysis of ADBE and CME in single nerve terminals Fluorescence-based approaches are highly informative in monitoring both ADBE and CME. However, the most direct assay to monitor both retrieval modes is to visualize the uptake of fluid phase markers into single nerve terminals by electron microscopy. HRP is a fluid phase marker that is taken up by both ADBE and CME during elevated neuronal activity (Evans and Cousin, 2007; Clayton et al., 2008). ADBE is detected as the appearance of large electron-dense endosome-like structures, whereas CME is detected as electron-dense small SVs. Both CME and ADBE were activated within the same nerve terminals after stimulation with 
A mCerulean
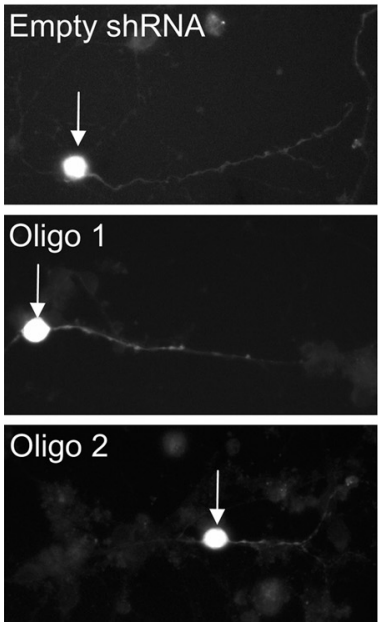

B mCerulean
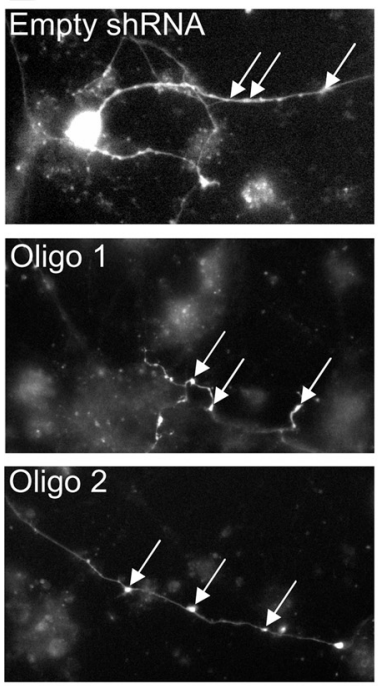

C

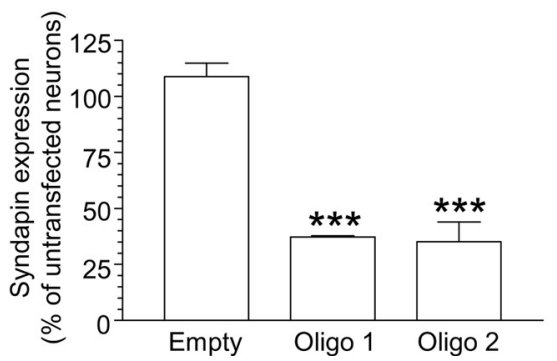

Syndapin
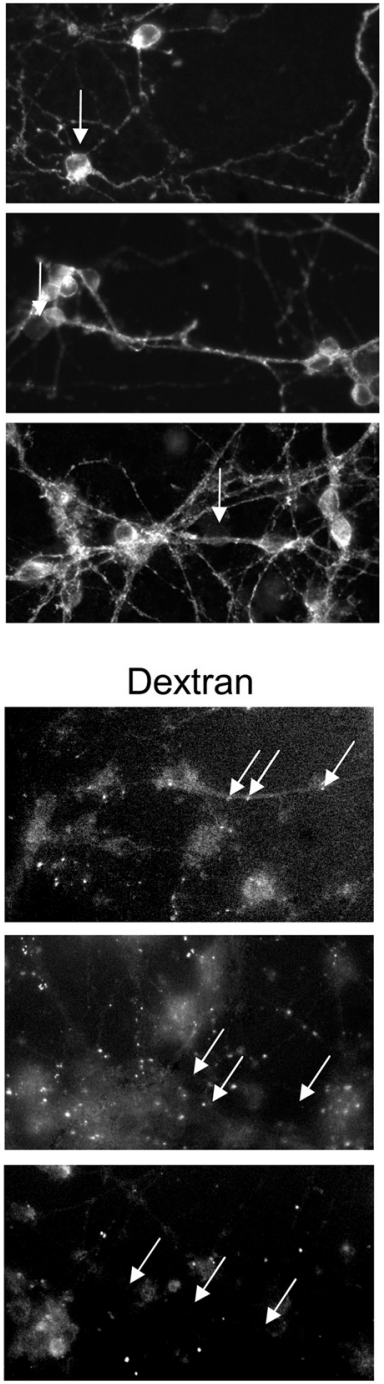

D

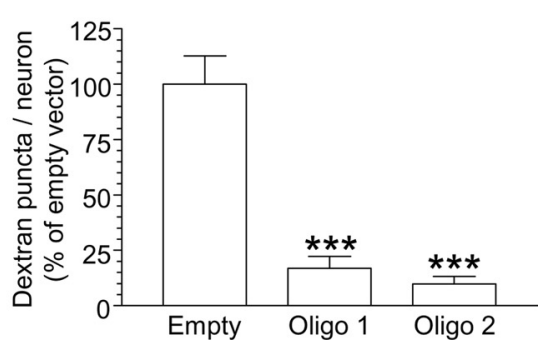

Figure 10. Silencing syndapin I expression inhibits ADBE. $A$, Granule neuron cultures were cotransfected with mCerulean and either shRNA against syndapin or empty shRNA vector. After $72 \mathrm{~h}$, syndapin expression was quantified using immunofluorescence. Left panels show transfected neurons with empty shRNA vector, Oligo 1, or Oligo 2. Middle panels show syndapin expression. Right panels show a merged image with transfected neuron in green and syndapin in red. Arrows highlight syndapin expression levels in transfected neurons. $\boldsymbol{B}$, Granule neuron cultures were transfected as in $\boldsymbol{A}$ and then incubated with $50 \mu \mathrm{m}$ tetramethylrhodamine-dextran. Dextran internalization was stimulated by a train of 800 action potentials ( $80 \mathrm{~Hz}$ ) followed by immediate washout. Left panels show transfected neurons with empty shRNA vector, 0ligo 1, or Oligo 2. Middle panels show dextran uptake. Right panels show a merged image with transfected neuron in green and dextran in red. Arrows highlight dextran uptake in transfected neurons. Note the dextran puncta in neurons transfected with empty vector, compared with the absence of puncta in either Oligo 1- or Oligo 2-transfected neurons. C, Quantification of syndapin knockdown in the cell body of shRNAexpressing neurons. (All $n=3$ experiments \pm SEM; ${ }^{* * *} p<0.001$, one-way ANOVA.) $\boldsymbol{D}$, Quantification of the effect of syndapin knockdown on dextran uptake in shRNA-expressing neurons. Results are expressed as dextran puncta per neuron, as a percentage of control (empty vector, $n=3$ experiments; 0 ligo 1 and 0 ligo 2, $n=4$; all \pm SEM, ${ }^{* * *} p<0.001$, one-way ANOVA).
$50 \mathrm{~mm} \mathrm{KCl}$ (Fig. 7A,D,E). The DynI ${ }_{769-}$ ${ }_{784} \mathrm{EE}$ phospho-box peptide did not affect the number of HRP-labeled endosomes or SVs (Fig. $7 B, D, E$ ). In contrast, the DynI $_{769-784}$ AA peptide greatly reduced the number of large-diameter, HRP-labeled endosomes but had little effect on the number of small-diameter, HRP-labeled SVs (Fig. 7C,D). This third approach independently confirms the requirement for the dynamin-syndapin interaction in ADBE previously observed with both FM1-43 and dextran approaches.

Neither phospho-box peptide affected the number of HRP-labeled SVs during intense stimulation, further supporting the conclusion that the dynamin-syndapin interaction does not participate in CME. To confirm this, we examined the effect of phospho-box peptides on HRP uptake evoked by mild neuronal activity (200 action potentials at $10 \mathrm{~Hz}$ ), where the dominant SV retrieval mode is CME. Neither peptide reduced the number of HRPlabeled SVs (Fig. 8), confirming that the activity-dependent dynamin-syndapin interaction plays no role in CME in central nerve terminals.

\section{Dynamin I GTPase activity is required for $\mathrm{ADBE}$}

We have shown that both dynamin I dephosphorylation and its interaction with syndapin are essential for ADBE but not CME. However, an obligate role for dynamin $\mathrm{I}$ in $\mathrm{ADBE}$ has been questioned in studies using the dynamin I knock-out mouse (Hayashi et al., 2008; Lou et al., 2008). We addressed this point by acutely inhibiting the GTPase activity of dynamin I and II with the pharmacological antagonist dynasore (Macia et al., 2006). This allowed a direct examination of the requirement for dynamin in ADBE without the complication of redundancy between different isoforms of dynamin.

The direct role of dynamin I in ADBE was first determined by monitoring dextran uptake during elevated neuronal activity (800 action potentials). Dynasore was used at concentrations $(80 \mu \mathrm{M})$ that were previously shown to eliminate SV endocytosis in neuronal cultures, but have no effect on SV exocytosis (Newton et al., 2006). Inhibition of dynamin GTPase activity reduced dextran uptake to $<20 \%$ of control, indicating an essential requirement for the enzyme in ADBE (Fig. 9A, $B$; control, $100 \pm 22.0$; dynasore, $16.0 \pm 2.7$; both $n=3, p=0.019$, Student's $t$ test).

Next, ADBE and CME were monitored in parallel by visualizing HRP uptake into single nerve terminals evoked by 800 ac- 

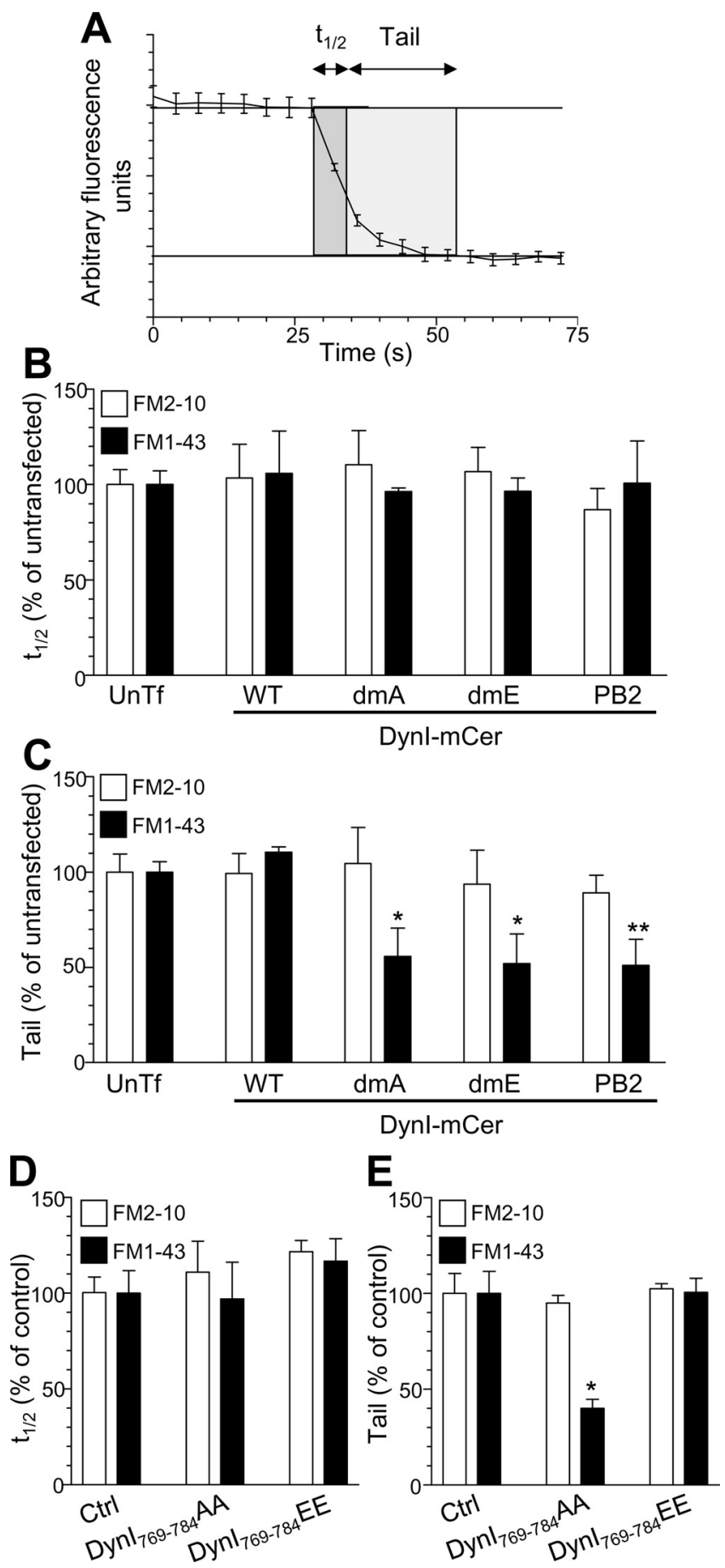

Figure 11. Inhibition of ADBE eliminates a sustained phase of SV exocytosis. $\boldsymbol{A}$, Two parameters were monitored with respect to dye unloading, the time for nerve terminals to lose $50 \%$ of their dye content ( $t_{1 / 2}$, shown in dark gray) and the time for the remainder to be unloaded (Tail, shown in light gray). In all experiments, dye was loaded with 800 action potentials $(80 \mathrm{~Hz})$ and washed away immediately after stimulation. Dye was unloaded with $50 \mathrm{~mm} \mathrm{KCl}$. B, C, Effect of overexpression of Dynl ${ }^{\text {WT }}$-mCer (WT) Dynl ${ }^{\mathrm{dmA}}$-mCer (dmA), Dynl ${ }^{\mathrm{dmE}}-\mathrm{mCer}(\mathrm{dmE})$, or Dynl ${ }^{\mathrm{PB} 2}$ mCer (PB2) on either the $t_{1 / 2}(\boldsymbol{B})$ or tail ( $C$ unloading kinetics of both FM2-10 (open bars) and FM1-43 (closed bars). Results are expressed as a percentage of untransfected neurons (FM1-43: Dynl ${ }^{\text {WT }}$-mCer, $n=3$; Dynl ${ }^{\mathrm{dmE}}$-mCer, $n=4$; Dynl ${ }^{\mathrm{dmA}}$-mCer, $n=5$, Dynl ${ }^{\mathrm{PB} 2}$-mCer, $n=3$; FM2-10: Dynl ${ }^{\text {WT }}$-mCer, $n=3$; Dynl ${ }^{\mathrm{dmE}}$-mCer, $n=4$; Dynl ${ }^{\mathrm{dmA}}$-mCer, $n=5$, Dynl ${ }^{\mathrm{PB} 2}$-mCer, $n=4 \pm$ SEM; one-way ANOVA, $\left.{ }^{*} p<0.05,{ }^{* *} p<0.01\right)$. D, E, Effect of Dynl ${ }_{769-784} \mathrm{AA}$ or Dynl $_{769-784}$ EE peptides on either the $t_{1 / 2}(\boldsymbol{D})$ or tail $(\boldsymbol{E})$ unloading kinetics of both FM2-10 (open bars) and FM1-43 (closed bars). Results are expressed as a percentage of control neurons [all $n=3 \pm$ SEM except FM1-43 control $(n=4)]$. One-way ANOVA, ${ }^{*} p<0.05$. tion potentials. Dynasore greatly reduced the number of HRPlabeled SVs, consistent with its essential role in CME (Fig. 8C,D; HRP-labeled SV s per nerve terminal, $1.62 \pm 0.12$; control, $0.60 \pm$ $0.08 ; n=127, p<0.0001$, Student's $t$ test). Dynasore also reduced the number of HRP-labeled endosomes by a similar extent, indicating that $\mathrm{ADBE}$ also requires dynamin GTPase activity (Fig. $8 C, D$; HRP-labeled endosomes per nerve terminal, $8.26 \pm 0.41$; control, $2.87 \pm 0.29 ; n=122, p<0.0001$, Student's $t$ test). The morphology of the HRP-labeled endosomes that were generated in the presence of dynasore was also unusual, with a large proportion forming elongated tubular structures rather than a typical spherical appearance (Fig. $8 \mathrm{E}$; malformed HRP endosomes as a percentage of total, 2.84\%; control, $20.29 \%$ dynasore). These malformations are indicative of a profound defect in the ADBE mode of endocytosis and are similar to structures previously observed in the nerve terminals of the dynamin I knock-out mouse (Ferguson et al., 2007; Hayashi et al., 2008). Therefore, both CME and ADBE have an essential requirement for dynamin GTPase activity, whereas the dephosphorylation of dynamin I is specifically required for $\mathrm{ADBE}$ and not $\mathrm{CME}$.

\section{Syndapin $\mathrm{I}$ is required for $\mathrm{ADBE}$}

The fact that dynamin I is required for both CME and ADBE suggests that syndapin I recruitment by dynamin I dephosphorylation may be the key step in the activation of ADBE. To test whether ADBE has an essential requirement for syndapin I, we silenced its expression using an shRNA vector system. Two independent vectors containing different oligonucleotide sequences greatly reduced syndapin I expression in our culture system, whereas the empty vector has no effect (Fig. 10A,C). We then examined the effect of syndapin I knockdown on dextran uptake, a selective marker of ADBE. Knockdown of syndapin I expression by both silencing vectors resulted in the near abolition of dextran uptake, whereas the empty vector has no effect (Fig. $10 \mathrm{~B}, \mathrm{D}$ ). Thus, there is an essential requirement for syndapin I in $\mathrm{ADBE}$, and its phosphorylation-dependent recruitment by dynamin $\mathrm{I}$ is a key step in this endocytosis mode.

\section{Reserve SV pool refilling is dependent on the} dynamin-syndapin interaction

A proposed physiological role of ADBE is the replenishment of the reserve pool of SVs (Richards et al., 2000). In agreement, we have shown that FM1-43, but not FM2-10, labels a pool of SVs that sustain neurotransmitter release during subsequent rounds of SV recycling (Evans and Cousin, 2007). When the unloading of both FM2-10 and FM1-43 was examined in control cultures in this study, the time taken for nerve terminals to lose the initial $50 \%$ of their dye content $\left(t_{1 / 2}\right)$ was equal (FM2$10,4.6 \pm 0.4 \mathrm{~s} ; \mathrm{FM} 1-43,4.6 \pm 0.5 \mathrm{~s} ; n=34$ experiments \pm SEM; not significant, Student's $t$ test). In contrast, the time taken to unload the remaining dye (tail; Fig. 11A) was significantly longer for FM1-43 compared with FM2-10 (FM2-10, $10.3 \pm 0.8 \mathrm{~s} ; \mathrm{FM} 1-43,18.2 \pm 1.0 \mathrm{~s} ; n=34$ experiments $\pm \mathrm{SEM}$, $p<0.001$, Student's $t$ test). Thus, FM1-43, but not FM2-10, labels an additional pool of SVs (most probably the reserve pool) that help to sustain neurotransmitter release (Evans and Cousin, 2007).

Because this sustained phase of FM1-43 unloading probably originates from $S V$-generated $A D B E$, we predicted that any perturbation of the dynamin-syndapin interaction should selectively eliminate this phase of release in a subsequent round of SV recycling. In agreement, the additional tail component 
of FM1-43 unloading was eliminated by either overexpression of the dominant-negative full-length dynamin mutants DynI $^{\mathrm{dmA}}-\mathrm{mCer}$, DynI ${ }^{\mathrm{dmE}}-\mathrm{mCer}$, and DynI ${ }^{\mathrm{PB} 2}-\mathrm{mCer}$ (Fig. $11 C$ ) or application of $\mathrm{DynI}_{769-784} \mathrm{AA}$ peptides in the previous loading phase (Fig. 11E). These treatments were specific for this sustained period of SV fusion, since they had no effects on either the tail component of FM2-10 (Fig. 11C,E) or on the $t_{1 / 2}$ for either FM1-43 or FM2-10 (Fig. $11 B, D$ ). Thus, the dynamin-syndapin interaction is essential for the replenishment of a reserve pool of SVs, highlighting the importance of ADBE in maintaining the fidelity of neurotransmission during periods of intense activity.

\section{Discussion}

Dynamin is required for CME, which is the main SV retrieval mode in nerve terminals at lower neuronal activity. We confirm these observations and show that the dynamin I phosphorylation-dephosphorylation cycle is not involved in CME of small synaptic vesicles. However dynamin mediates multiple endocytic modes in nerve terminals, and the identity of the mode(s) specifically controlled by dynamin I dephosphorylation was unknown. We now show that the dephosphorylation of dynamin I is activity dependent and has an activation threshold that is closely tuned to the stimulation conditions that recruit a second endocytic mode, ADBE. This dephosphorylation event and the resulting downstream interaction with syndapin do not play a role in CME. Rather, they are both essential for the recruitment of ADBE in nerve terminals. The recruitment of ADBE during periods of higher neuronal activity provides nerve terminals with a second high-capacity endocytic mode alongside CME. Evidence for this is as follows: (1) dynamin I is dephosphorylated by levels of neuronal activity that trigger ADBE, but not activity that evokes CME; (2) overexpression of phospho-site dynamin I mutants arrest only ADBE; (3) perturbation of the dynamin-syndapin interaction using either overexpression of syndapin interaction mutants or delivery of competitive phospho-box peptides blocks ADBE but not CME using multiple independent assays in both central nerve terminals and non-neuronal cells.

\section{Dynamin I dephosphorylation is essential for ADBE}

$\mathrm{ADBE}$ is triggered by increased neuronal activity in central nerve terminals (Clayton et al., 2008). Calcium influx is the likely trigger, since this will provide an accurate report of both the pattern and intensity of stimulation. In agreement, calcium influx driven by increased neuronal activity increases the rate of endocytosis in different neuronal systems ( $\mathrm{Wu}$ et al., 2005; Balaji et al., 2008). Calcineurin is an excellent sensor for detecting increased calcium influx during high activity, because of its cytosolic location and low micromolar affinity for calcium (Klee et al., 1979). Indeed, studies in different systems have shown a requirement for calcineurin in endocytosis during elevated, but not mild neuronal activity (Chan and Smith, 2001; Kumashiro et al., 2005). We now show that the calcineurin-dependent dephosphorylation of dynamin I has an activation threshold that closely correlates with the levels of neuronal activity that trigger ADBE, further confirming its essential role in the process (Evans and Cousin, 2007). It will be of importance to determine whether other dephosphins are also dephosphorylated in an activity-dependent manner by calcineurin and whether these events will also be selective for the ADBE mode.

Since activity-dependent dephosphorylation of dynamin I is essential for the triggering of ADBE, it infers that dynamin I itself is essential for the process. Studies using the dynamin I knock-out mouse have provided evidence both for and against this role. In support, endocytosis during mild neuronal activity (mediated by CME) is unaffected by the absence of dynamin I in all nerve terminals studied to date (Ferguson et al., 2007; Lou et al., 2008). Furthermore, typical small central nerve terminals derived from these mice display a SV endocytosis defect that is only apparent during, but not after, action potential trains of elevated frequency (Ferguson et al., 2007). Since ADBE is the dominant SV retrieval mode during elevated neuronal activity, this implicates dynamin I in the process. However a different study examining inhibitory nerve terminals showed that endosomal labeling by HRP still occurs during prolonged stimulation with elevated $\mathrm{KCl}$ (Hayashi et al., 2008), suggesting that dynamin I may not be essential for ADBE. Furthermore, atypical large glutamatergic nerve terminals derived from dynamin I knock-out mice appear to show a requirement for dynamin I during slow poststimulation CME (Lou et al., 2008), rather than during stimulation as seen in small nerve terminals (Ferguson et al., 2007). It is most probable that the contradictory evidence originating from these studies is attributable to compensation from other dynamin isoforms such as dynamin III, which is also dephosphorylated at similar sites on nerve terminal stimulation (Graham et al., 2007). Because of this, we determined the role of dynamin in ADBE by acutely inhibiting its activity with the antagonist dynasore during action potential stimulation (Macia et al., 2006; Newton et al., 2006). Dynamin GTPase inhibition caused a clear reduction in both dextran uptake and HRP labeling of endosomes, indicative of a key role for dynamin GTPase activity in ADBE. Additionally, a dramatic change in endosome morphology was also observed, suggesting that a residual component of $\mathrm{ADBE}$ was grossly affected by dynamin GTPase inhibition. Thus, dynamin I activity is required for both $\mathrm{ADBE}$ and CME, but its dephosphorylation is only essential for ADBE.

\section{The phosphorylation-dependent dynamin-syndapin interaction is essential for ADBE}

Our results show that the phospho-dependent dynamin-syndapin interaction is essential for ADBE, using both inhibitory peptides and overexpression of syndapin interaction mutants. Importantly, both approaches do not interfere with the binding of other proteins to dynamin I such as amphiphysin and endophilin (Anggono et al., 2006; Anggono and Robinson, 2007). Syndapin differs from both amphiphysin and endophilin in that it has an F-BAR domain at its $\mathrm{N}$ terminus, whereas the other two proteins have an N-BAR domain (Peter et al., 2004; Itoh et al., 2005). F-BAR domains interact with membranes of more shallow curvature than N-BAR domains (Henne et al., 2007; Shimada et al., 2007). This provides a potential molecular explanation for the selective requirement for the dynamin-syndapin interaction in $\mathrm{ADBE}$, since the F-BAR domain of syndapin will support the invagination and tubulation of large endosomes (which have shallow curvature), whereas the N-BAR domains of both amphiphysin and endophilin will facilitate the invagination of more tightly curved SVs. In agreement, recent studies in lamprey neurons found an activity-dependent requirement for syndapin in SV endocytosis (Andersson et al., 2008). Furthermore, ADBE was perturbed by injection of anti-syndapin antibodies, whereas CME was unaffected (Andersson et al., 2008). Thus, ADBE has an essential requirement for syndapin (as seen in our shRNA stud- 
ies), which is recruited by the activity-dependent dephosphorylation of dynamin I.

\section{Multiple SV endocytosis modes are differentially regulated by dynamin I dephosphorylation}

We have shown through three independent approaches that the dynamin-syndapin interaction has no role in CME (despite the fact that dynamin itself is important). First, inhibition of the interaction with phospho-box peptides does not interfere with uptake of FM1-43 during mild stimulation, a protocol that only activates CME (Granseth et al., 2006; Evans and Cousin, 2007; Clayton et al., 2008). Second, phospho-box peptides do not inhibit generation of HRP-labeled SVs evoked by either elevated or mild neuronal activity, in contrast to their effect on HRP labeling of endosomes. Finally, phospho-box peptides do not inhibit the internalization of transferrin receptors through a classical clathrin-dependent pathway in non-neuronal cells. Thus, the phospho-dependent dynaminsyndapin interaction is specifically required for $\mathrm{ADBE}$ and not CME.

These results provide a unifying hypothesis of how neuronal activity controls the molecular interactions that determine the activation of SV retrieval modes in central nerve terminals. Both CME and ADBE have an obligate requirement for dynamin I GTPase activity. During mild neuronal activity the major membrane retrieval mode is CME (Granseth et al., 2006; Clayton et al., 2008). Dynamin I is not dephosphorylated by these stimulation conditions; therefore, ADBE is not activated. For CME to proceed in neurons, an essential interaction between dynamin I and the N-BAR protein amphiphysin has to occur (Shupliakov et al., 1997; Jockusch et al., 2005). Dynamin I binds amphiphysin regardless of its phosphorylation status (Tan et al., 2003; Anggono et al., 2006; Graham et al., 2007), potentially explaining why CME proceeds independently of the level of neuronal activity. Thus, CME is dynamin dependent but is not dependent on dynamin I dephosphorylation. When nerve terminals are challenged by elevated neuronal activity, the activity-dependent increase in calcium influx stimulates calcineurin to dephosphorylate dynamin I. This dephosphorylation event then stimulates the interaction of dynamin I with the F-BAR protein syndapin to mediate ADBE. Thus, we have established the molecular cascade responsible for the triggering of ADBE in central nerve terminals, with the dephosphorylation of dynamin I being the key determinant in the activity-dependent recruitment of an additional SV retrieval mode.

\section{References}

Andersson F, Jakobsson J, Löw P, Shupliakov O, Brodin L (2008) Perturbation of syndapin/PACSIN impairs synaptic vesicle recycling evoked by intense stimulation. J Neurosci 28:3925-3933.

Anggono V, Robinson PJ (2007) Syndapin I and endophilin I bind overlapping proline-rich regions of dynamin I: role in synaptic vesicle endocytosis. J Neurochem 102:931-943.

Anggono V, Smillie KJ, Graham ME, Valova VA, Cousin MA, Robinson PJ (2006) Syndapin I is the phosphorylation-regulated dynamin I partner in synaptic vesicle endocytosis. Nat Neurosci 9:752-760.

Balaji J, Armbruster M, Ryan TA (2008) Calcium control of endocytic capacity at a CNS synapse. J Neurosci 28:6742-6749.

Chan SA, Smith C (2001) Physiological stimuli evoke two forms of endocytosis in bovine chromaffin cells. J Physiol 537:871-885.

Clayton EL, Cousin MA (2008) Differential labelling of bulk endocytosis in nerve terminals by FM dyes. Neurochem Int 53:51-55.

Clayton EL, Evans GJ, Cousin MA (2008) Bulk synaptic vesicle endocytosis is rapidly triggered during strong stimulation. J Neurosci 28:6627-6632.
Cousin MA, Robinson PJ (2001) The dephosphins: dephosphorylation by calcineurin triggers synaptic vesicle endocytosis. Trends Neurosci 24:659-665.

Cousin MA, Malladi CS, Tan TC, Raymond CR, Smillie KJ, Robinson PJ (2003) Synapsin I-associated phosphatidylinositol 3-kinase mediates synaptic vesicle delivery to the readily releasable pool. J Biol Chem 278:29065-29071.

Evans GJ, Cousin MA (2007) Activity-dependent control of slow synaptic vesicle endocytosis by cyclin-dependent kinase 5. J Neurosci 27:401-411.

Ferguson SM, Brasnjo G, Hayashi M, Wölfel M, Collesi C, Giovedi S, Raimondi A, Gong LW, Ariel P, Paradise S, O'toole E, Flavell R, Cremona O, Miesenböck G, Ryan TA, De Camilli P (2007) A selective activitydependent requirement for dynamin 1 in synaptic vesicle endocytosis. Science 316:570-574.

Grabs D, Slepnev VI, Songyang Z, David C, Lynch M, Cantley LC, De Camilli P (1997) The SH3 domain of amphiphysin binds the proline-rich domain of dynamin at a single site that defines a new $\mathrm{SH} 3$ binding consensus sequence. J Biol Chem 272:13419-13425.

Graham ME, Anggono V, Bache N, Larsen MR, Craft GE, Robinson PJ (2007) The in vivo phosphorylation sites of rat brain dynamin I. J Biol Chem 282:14695-14707.

Granseth B, Odermatt B, Royle SJ, Lagnado L (2006) Clathrin-mediated endocytosis is the dominant mechanism of vesicle retrieval at hippocampal synapses. Neuron 51:773-786.

Hayashi M, Raimondi A, O’Toole E, Paradise S, Collesi C, Cremona O, Ferguson SM, De Camilli P (2008) Cell- and stimulus-dependent heterogeneity of synaptic vesicle endocytic recycling mechanisms revealed by studies of dynamin 1-null neurons. Proc Natl Acad Sci U S A 105:2175-2180.

Henne WM, Kent HM, Ford MG, Hegde BG, Daumke O, Butler PJ, Mittal R, Langen R, Evans PR, McMahon HT (2007) Structure and analysis of FCHo2 F-BAR domain: a dimerizing and membrane recruitment module that effects membrane curvature. Structure 15:839-852.

Holt M, Cooke A, Wu MM, Lagnado L (2003) Bulk membrane retrieval in the synaptic terminal of retinal bipolar cells. J Neurosci 23:1329-1339.

Itoh T, Erdmann KS, Roux A, Habermann B, Werner H, De Camilli P (2005) Dynamin and the actin cytoskeleton cooperatively regulate plasma membrane invagination by BAR and F-BAR proteins. Dev Cell 9:791-804.

Jockusch WJ, Praefcke GJ, McMahon HT, Lagnado L (2005) Clathrindependent and clathrin-independent retrieval of synaptic vesicles in retinal bipolar cells. Neuron 46:869-878.

Klee CB, Crouch TH, Krinks MH (1979) Calcineurin: a calcium- and calmodulin-binding protein of the nervous system. Proc Natl Acad Sci U S A 76:6270-6273.

Kumashiro S, Lu YF, Tomizawa K, Matsushita M, Wei FY, Matsui H (2005) Regulation of synaptic vesicle recycling by calcineurin in different vesicle pools. Neurosci Res 51:435-443.

Lee SY, Voronov S, Letinic K, Nairn AC, Di Paolo G, De Camilli P (2005) Regulation of the interaction between PIPKI gamma and talin by prolinedirected protein kinases. J Cell Biol 168:789-799.

Lou X, Paradise S, Ferguson SM, De Camilli P (2008) Selective saturation of slow endocytosis at a giant glutamatergic central synapse lacking dynamin 1. Proc Natl Acad Sci U S A 105:17555-17560.

Macia E, Ehrlich M, Massol R, Boucrot E, Brunner C, Kirchhausen T (2006) Dynasore, a cell-permeable inhibitor of dynamin. Dev Cell 10:839-850.

Marks B, Stowell MH, Vallis Y, Mills IG, Gibson A, Hopkins CR, McMahon HT (2001) GTPase activity of dynamin and resulting conformation change are essential for endocytosis. Nature 410:231-235.

Newton AJ, Kirchhausen T, Murthy VN (2006) Inhibition of dynamin completely blocks compensatory synaptic vesicle endocytosis. Proc Natl Acad Sci U S A 103:17955-17960.

Peter BJ, Kent HM, Mills IG, Vallis Y, Butler PJ, Evans PR, McMahon HT (2004) BAR domains as sensors of membrane curvature: the amphiphysin BAR structure. Science 303:495-499.

Qualmann B, Roos J, DiGregorio PJ, Kelly RB (1999) Syndapin I, a synaptic dynamin-binding protein that associates with the neural Wiskott-Aldrich syndrome protein. Mol Biol Cell 10:501-513.

Richards DA, Guatimosim C, Betz WJ (2000) Two endocytic recycling routes selectively fill two vesicle pools in frog motor nerve terminals. Neuron 27:551-559.

Ringstad N, Nemoto Y, De Camilli P (1997) The SH3p4/Sh3p8/SH3p13 
protein family: binding partners for synaptojanin and dynamin via a Grb2-like Src homology 3 domain. Proc Natl Acad Sci USA 94:8569-8574.

Rizzo MA, Springer GH, Granada B, Piston DW (2004) An improved cyan fluorescent protein variant useful for FRET. Nat Biotechnol 22:445-449.

Rizzoli SO, Betz WJ (2005) Synaptic vesicle pools. Nat Rev Neurosci 6:57-69.

Royle SJ, Lagnado L (2003) Endocytosis at the synaptic terminal. J Physiol 553:345-355.

Shimada A, Niwa H, Tsujita K, Suetsugu S, Nitta K, Hanawa-Suetsugu K, Akasaka R, Nishino Y, Toyama M, Chen L, Liu ZJ, Wang BC, Yamamoto M, Terada T, Miyazawa A, Tanaka A, Sugano S, Shirouzu M, Nagayama K, Takenawa T, et al. (2007) Curved EFC/F-BAR-domain dimers are joined end to end into a filament for membrane invagination in endocytosis. Cell 129:761-772.
Shupliakov O, Löw P, Grabs D, Gad H, Chen H, David C, Takei K, De Camilli P, Brodin L (1997) Synaptic vesicle endocytosis impaired by disruption of dynamin-SH3 domain interactions. Science 276:259-263.

Tan TC, Valova VA, Malladi CS, Graham ME, Berven LA, Jupp OJ, Hansra G, McClure SJ, Sarcevic B, Boadle RA, Larsen MR, Cousin MA, Robinson PJ (2003) Cdk5 is essential for synaptic vesicle endocytosis. Nat Cell Biol 5:701-710.

Teng H, Lin MY, Wilkinson RS (2007) Macroendocytosis and endosome processing in snake motor boutons. J Physiol 582:243-262.

Wu LG, Ryan TA, Lagnado L (2007) Modes of vesicle retrieval at ribbon synapses, calyx-type synapses, and small central synapses. J Neurosci 27:11793-11802.

Wu W, Xu J, Wu XS, Wu LG (2005) Activity-dependent acceleration of endocytosis at a central synapse. J Neurosci 25:11676-11683. 